\title{
Mandenkan
}

MANDENIKAN Bulletin semestriel d'études linguistiques mandé

49 | 2013

Le maninka du Niokolo

\section{Les numéraux et l'expression de la quantité}

Section 12

\section{Denis Creissels}

\section{(2) OpenEdition}

Journals

Édition électronique

URL : https://journals.openedition.org/mandenkan/608

DOI : 10.4000/mandenkan.608

ISSN : 2104-371X

Éditeur

Llacan UMR 8135 CNRS/Inalco

Édition imprimée

Date de publication : 1 juin 2013

Pagination : 88-93

ISSN : 0752-5443

Référence électronique

Denis Creissels, "Les numéraux et l'expression de la quantité », Mandenkan [En ligne], 49 | 2013, mis en ligne le 25 avril 2014, consulté le 16 janvier 2023. URL : http://journals.openedition.org/ mandenkan/608; DOI : https://doi.org/10.4000/mandenkan.608

Ce document a été généré automatiquement le 16 janvier 2023.

\section{(c) $)(1)(2)$}

Creative Commons - Attribution - Pas d'Utilisation Commerciale - Partage dans les Mêmes Conditions 4.0 International - CC BY-NC-SA 4.0

https://creativecommons.org/licenses/by-nc-sa/4.0/ 


\section{Les numéraux et l'expression de la quantité}

Section 12

Denis Creissels

\subsection{Le système de numération}

\subsubsection{Numéraux élémentaires}

1 L'inventaire des numéraux élémentaires est le suivant :

\begin{tabular}{|l|l|l|}
\hline & kilin' & 'un' \\
\hline fúla & 'deux' \\
\hline sába & 'trois' \\
\hline naani' & 'quatre' \\
\hline luulu' & 'cinq' \\
\hline wooro' & 'six' \\
\hline worowúla & 'sept' \\
\hline seyi' & 'huit' \\
\hline kónonto' & 'neuf' \\
\hline tay' & 'dix' \\
\hline mu'-́yay & 'vingt' \\
\hline
\end{tabular}




\begin{tabular}{|l|l|l|}
\hline & ke'-ime & 'cent' \\
\hline & wuli' waa & 'mille' \\
\hline
\end{tabular}

2 Il faut toutefois noter que parmi ces termes, wuli' et waa 'mille' n'apparaissent jamais seuls. A la différence de kéme, qui isolé a la valeur numérique 100, wuli' et waa 'mille' doivent se combiner à kiliy 'un' pour exprimer la valeur numérique 1000 :

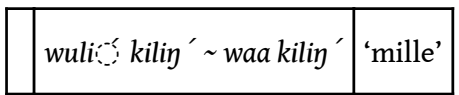

Wuli' et waa semblent également usuels.

\subsubsection{Dizaines, centaines et milliers}

Les termes pour les dizaines de 30 à 90 sont formés de manière équivalente avec comme premier formant ou bien tag ' (qui s'utilise isolément avec la valeur numérique 10) ou bien bii' (qui ne s'utilise pas isolément). La simple juxtaposition du deuxième formant exprime la multiplication.

\begin{tabular}{|c|c|c|}
\hline bii sába & $\sim$ tay sába & 'trente' \\
\hline bi & $\sim$ ta & 'quarante' \\
\hline bi $i^{-}{ }_{i}$ l luulu' & $\sim$ ta & 'cinquante' \\
\hline$b i$ & $\sim$ ta & 'soixante' \\
\hline bi $i$ worowúla & $\sim$ ta & 'soixante-dix' \\
\hline$b i_{-}^{-} i$ seyi $^{\prime}$ & $\sim$ tai-y seyi & 'quatre-vingts' \\
\hline bii kónonto' & tạ kónonto' & 'quatre-vingt-dix' \\
\hline
\end{tabular}

$5 \quad$ Les termes pour les centaines à partir de deux cents et pour les milliers sont formés de manière analogue (postposition du multiplicateur au multiplicande) :

\begin{tabular}{|l|l|l|l|}
\hline & kéme fúla & 'deux cents' & \\
\hline & ke'́me sa'́ba & 'trois cents' & \\
\hline & ke'́me naani' & 'quatre cents & \\
\hline & ke'-ime luulu' & 'cinq cents' & \\
\hline & etc. & & \\
\hline
\end{tabular}




\begin{tabular}{|c|c|c|}
\hline waa fúla & wuli fúla & 'deux mille' \\
\hline waa saíba & wuli sa & 'trois mille' \\
\hline wa'a naani' & 〜 wulis naani' & 'quatre mille' \\
\hline wa: aluulu' & 〜 wuli & 'cinq mille' \\
\hline etc. & & \\
\hline
\end{tabular}

6 On observe que la différence tonale entre wuli ' et waa, apparente en combinaison avec kiliy', est ici neutralisée, ce qui est à mettre en relation avec le fait les numéraux simples à partir de deux ont un comportement tonal particulier relativement au nom qu'ils modifient: le ton modulé de wa ans wa naani', wa a luulu' etc. n'est pas dû à un ton flottant haut final (dont ce terme est dépourvu) mais à une propriété tonale particulière des numéraux auxquels il se combine (cf. 12.2).

\subsubsection{Autres}

$7 \quad$ Les autres nombres sont formés par addition, l'addition étant encodée par nin a-́nin

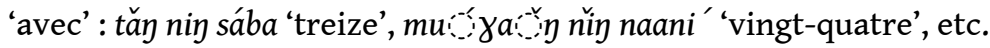

\subsection{Les numéraux cardinaux comme modifieurs de noms}

\subsubsection{Observations générales}

Les numéraux se postposent aux noms qu'ils modifient, et comme c'est de manière générale le cas en mandingue, en présence d'un numéral, le marqueur de détermination est syntaxiquement facultatif. Le marqueur de détermination, qui s'attache au numéral, implique une valeur de défini. Il est usuellement suivi du marqueur de pluriel, qui n'apparaît par contre pas en l'absence du marqueur de détermination.

\begin{tabular}{|c|c|c|c|c|c|c|c|c|c|}
\hline (127) & a. & Dindiy & $s a \vdots b a$ & $n a-a-t a$. & b. & Dindiy & $s a a^{\prime-1} b a$ & lu & na $a-t a$. \\
\hline & & enfant & trois & venir-ACPP & & enfant & trois.D & PL & venir-ACPP \\
\hline & & \multicolumn{3}{|c|}{ 'Trois enfants sont venus.' } & \multicolumn{5}{|c|}{ 'Les trois enfants sont venus.' } \\
\hline
\end{tabular}

\begin{tabular}{|c|c|c|c|c|c|c|c|c|}
\hline c. & Dindi & naani & na: $a-t a$. & d. & Dindi: & naan-o:-o & lu & na $a-t a$. \\
\hline & enfant & quatre & venir-ACPP & & enfant & quatre-D & PL & venir-ACPP \\
\hline & \multicolumn{3}{|c|}{ 'Quatre enfants sont venus.' } & & \multicolumn{4}{|c|}{ 'Les quatre enfants sont venus.' } \\
\hline
\end{tabular}


12.2.2. Kilin' 'un'

9 A la différence des autres numéraux simples (cf. 12.2.3), avec kilin', il n'apparaitt jamais de ton haut sur la syllabe finale du nom qui précède kilin' :

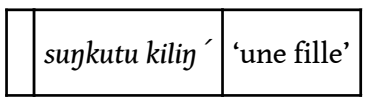

10 Kiliy' en fonction de modifieur forme avec le nom qu'il modifie un syntagme dans lequel la tonalité du premier terme est simplement modifiée comme elle l'est de manière générale dans les syntagmes à compacité tonale (perte d'un éventuel ton haut non-initial).

11 Il y a des raisons de penser que kilin' était à l'origine un qualifiant ('seul', 'isolé') qui formait avec le nom un syntagme à compacité tonale totale dans lequel il a été réanalysé comme numéral. Ce qui suggère cette analyse, c'est notamment le fait qu'à la différence des autres numéraux, kilin' peut prendre le suffixe d'abstraction -yaa pour donner le verbe kílinyaa 'être unique, isolé' :

\begin{tabular}{|l|l|l|l|l|l|}
\hline (128) & Kilin-yáa & bén-ta & Ala & lée & ma. \\
\hline & un-abstr.d & convenir-ACPP & Dieu & foc & OBL \\
\hline & 'Dieu seul est unique.' litt. 'L'unicité n'est compatible qu'avec Dieu.' \\
\hline
\end{tabular}

12 En outre, le fait que kíliyyaa ait un ton initial haut suggère que l'absence de ton initial haut dans kilin' s'explique par la réanalyse d'une construction à compacité tonale totale.

\subsubsection{Autres numéraux simples}

13 Comme cela a déjà été exposé en 3.7.1.4, avec les autres numéraux simples, un ton haut apparaît sur la syllabe finale du mot précédent dans les mêmes conditions qu'à une limite de mots ordinaires, mais il convient tout de même de considérer que cette construction est à compacité tonale partielle, car les noms de schème tonal noncanonique perdent leurs tons hauts non-initiaux.

\subsubsection{Dizaines, centaines, milliers}

14 Avec les numéraux complexes exprimant des multiples de dix, cent ou mille, on peut résumer les observations en posant que ces numéraux forment avec le nom qu'ils modifient un syntagme présentant le même type de compacité tonale partielle que la construction 'nom + qualifiant dérivé' (cf. 3.7.1.3). En effet le ton de ces numéraux reste identique à celui observé en isolation, tandis que le nom qui les précède ne présente jamais le ton haut démarcatif, et perd son ton haut non-initial s'il s'agit d'un nom de schème tonal non-canonique : 


\begin{tabular}{|c|c|c|}
\hline suykutu bii sába & sugkutu tay sába & 'trente filles' \\
\hline suykutu bíi naani' & suykutu táy naani & 'quarante filles' \\
\hline suykutu ke-ime fúla & 'deux cents filles' & \\
\hline suykutu ke-ime naani & 'quatre cents filles' & \\
\hline kuluy wuli kilin' & kuluy waa kilig' & 'mille bateaux' \\
\hline kuluy wuli fúla & kuluy waa fui & 'deux mille bateaux' \\
\hline
\end{tabular}

\subsection{Les ordinaux}

En dehors de foloo 'premier', qui n'a aucune parenté avec kiliy 'un' mais qui s'emploie par ailleurs comme verbe au sens de 'commencer'1, les ordinaux sont dérivés des numéraux cardinaux par adjonction du suffixe -ñjay. Syntaxiquement, ils s'emploient comme modifieurs de noms dans la construction qualificative décrite en 13, mais aussi comme verbes intransitifs ('se produire une énième fois') et comme verbes transitifs ('faire quelque chose une énième fois').

\begin{tabular}{|l|l|l|l|l|}
\hline$(129)$ & a. & $\tilde{N}$ ì & kanáa & fúla-ñjay! \\
\hline & & dem & sUBJN & deux-ORD \\
\hline & \multicolumn{3}{|c|}{ 'Que ceci ne se reproduise pas!' } \\
\hline
\end{tabular}

\begin{tabular}{|l|l|l|l|l|}
\hline & b. & Kanáa & ñiy & fúla-ñjay ! \\
\hline & & subjn & DEM & deux-ORD \\
\hline & & \multicolumn{3}{|c|}{ 'Ne refais pas ceci !' } \\
\hline
\end{tabular}

\subsection{Quantifieurs divers}

Le questionnement sur le dénombrement ou la quantité peut se faire au moyen de jálu 'combien ?', qui se postpose de la même façon qu'un numéral au nom qu'il modifie.

\begin{tabular}{|l|l|l|l|l|l|}
\hline$(130)$ & a. & Móyo & jálu & be & ján? \\
\hline & & personne & combien & Coploc & ici \\
\hline & & \multicolumn{4}{|c|}{ 'Combien de personnes y a-t-il ici ?’ } \\
\hline
\end{tabular}




\begin{tabular}{|l|l|l|l|l|l|l|}
\hline & b. & I & yé & yeje & jálu & múta? \\
\hline & & 2 2sg & ACPP & poisson & combien & attraper \\
\hline & & \multicolumn{4}{|c|}{ 'Combien de poissons as-tu attrapés ?’ } \\
\hline
\end{tabular}

17 Avec les noms de substances mesurables mais non dénombrables, ‘combien ?' peut aussi

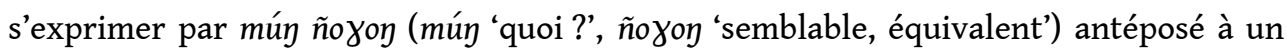
nom à la forme déterminée.

\begin{tabular}{|c|c|c|c|c|c|}
\hline (131) & a. & Mún & 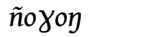 & máalóo & $t u-t a ?$ \\
\hline & & quoi & équivalent & riz-D & rester-ACРP \\
\hline & & \multicolumn{4}{|c|}{ 'Quelle quantité de riz est restée ?' } \\
\hline
\end{tabular}

\begin{tabular}{|l|l|l|l|l|l|l|l|}
\hline & b. & I & ye & múy & noyón & jiy-o & náati ? \\
\hline & & $2 s g$ & ACPP & quoi & équivalent & eau-D & apporter \\
\hline & \multicolumn{6}{|c|}{ 'Quelle quantité d'eau as-tu apporté ?' } \\
\hline
\end{tabular}

18 'Beaucoup' peut s'exprimer en postposant jamáa au nom à la forme nue. Jámaa est par ailleurs un nom signifiant 'foule', et le ton du syntagme formé par jamáa 'beaucoup' avec le nom qui le précède s'explique par cette étymologie. On peut en effet le prédire en considérant que tout se passe comme si on avait là un nom composé 'une foule de N' à la forme définie.

\begin{tabular}{|l|l|l|l|l|l|l|l|l|}
\hline (132) & Kóno & jamáa & lée & se & i & lóo & bántáy-o & to. \\
\hline & oiseau & beaucoup & foc & potp & REFL & dresser & fromager-D & LOC \\
\hline & 'Beaucoup d'oiseaux se posent sur le fromager.' \\
\hline
\end{tabular}

19 Avec les noms de substances mesurables mais non dénombrables, le suffixe diminutif -ndiy peut prendre la valeur de 'un peu de', et le suffixe augmentatif -baa peut prendre la valeur de 'beaucoup'. Dans cette valeur, -baa a une variante facultative -bumbaa.

\begin{tabular}{|l|l|l|l|l|l|l|}
\hline (133) & a. & Á & ye & tíya-báa & lée & sóto. \\
\hline & & $3 s g$ & ACPP & arachide-AUGM & foc & obtenir \\
\hline & & \multicolumn{3}{|c|}{ 'Il a récolté beaucoup d'arachide.' } \\
\hline
\end{tabular}




\begin{tabular}{|l|l|l|l|l|}
\hline & b. & Jii-báa & lée & náa-ta. \\
\hline & & eau-augm & FOc & venir-ACPP \\
\hline & & \multicolumn{2}{|c|}{ 'Il a beaucoup plu.' } \\
\hline
\end{tabular}

\begin{tabular}{|l|l|l|l|l|}
\hline & c. & T'íya-bumbáa & lée & sóto-ta. \\
\hline & & arachide-AUGM & FOC & obtenir-ACPP \\
\hline & & \multicolumn{2}{|l|}{ 'On a récolté beaucoup d'arachide.' } \\
\hline
\end{tabular}

'Beaucoup' peut aussi s'exprimer au moyen de siyaamay (dérivé étymologiquement de siyaa 'être abondant'), qui s'utilise aussi bien pour des quantités dénombrables ou non dénombrables. Siyaamay se construit comme un numéral: il succède à un nom à la forme nue, et ne porte pas lui-même la marque de détermination nominale. Avec un nom comme suykutu 'fille', on voit qu'il s'agit d'un syntagme à compacité tonale. En effet, dans le cas contraire, le ton haut démarcatif devrait apparaître sur la syllabe finale de sugkutu.

\begin{tabular}{|l|l|l|l|l|l|}
\hline (134) & a. & Tíya & siyaamay & née & sóto-ta. \\
\hline & & arachide & beaucoup & FOC & obtenir-ACPP \\
\hline & \multicolumn{3}{|l}{ 'On a récolté beaucoup d'arachide.' } \\
\hline
\end{tabular}

\begin{tabular}{|l|l|l|l|l|l|l|}
\hline & b. & Móyo & siyaamay & née & be & ján. \\
\hline & & personne & beaucoup & foc & coploc & ici \\
\hline & & \multicolumn{3}{|c|}{ 'Il y a beaucoup de gens ici.' } \\
\hline
\end{tabular}

\begin{tabular}{|l|l|l|l|l|l|}
\hline & c. & Suykutu & siyaamay & née & naía-ta. \\
\hline & & fille & beaucoup & foc & venir-ACPP \\
\hline & & \multicolumn{4}{|c|}{ 'Beaucoup de filles sont venues.' } \\
\hline
\end{tabular}

'Quelques-uns' peut s'exprimer au moyen de dantay, qui se construit aussi comme un numéral, et qui forme avec le nom un syntagme à compacité tonale.

\begin{tabular}{|l|l|l|l|l|l|l|}
\hline (135) & Supkutu & dantán & tu-ta & lée & sáatée & kono. \\
\hline & fille & quelques & rester-ACPP & Foc & village.D & dans \\
\hline
\end{tabular}


'Il est resté quelques filles au village.'

22

'Un peu' par référence à une substance mesurable mais non dénombrable peut s'exprimer au moyen de dondin précédé d'un nom à la forme déterminée.

\begin{tabular}{|l|l|l|l|l|l|l|}
\hline (136) & a. & Naajíy-o & dondiy & $k e$ & $\eta$ & kún! \\
\hline & & sauce-D & un_peu & mettre & 1 sg & SPHP \\
\hline & \multicolumn{4}{|l}{} \\
\hline
\end{tabular}

\begin{tabular}{|l|l|l|l|l|}
\hline & b. & Naajiy-o & dondin & na'-iati! \\
\hline & & sauce-d & un_peu & apporter \\
\hline & & \multicolumn{3}{|c|}{ 'Mets-moi un peu de sauce!' } \\
\hline
\end{tabular}

\begin{tabular}{|c|c|c|c|c|c|c|c|}
\hline c. & I & la & tíya & dondiy & $d i i_{-}^{-} i$ & $y$ & $m a !$ \\
\hline & $2 \mathrm{sg}$ & GEN & arachide.D & un_peu & donner & $1 \mathrm{sg}$ & obl \\
\hline & 'D & & & $e$ & aides!' & & \\
\hline
\end{tabular}

\begin{tabular}{|l|l|l|l|l|l|l|}
\hline & d. & I & la & tiya & dondin & ma _ŕra! \\
\hline & & $2 s g$ & GEN & arachide.D & un_peu & garder \\
\hline & & \multicolumn{5}{|c|}{ Mets de côté un peu de tes arachides!' } \\
\hline
\end{tabular}

Enfin avec les termes exprimant une mesure, on a la construction illustrée en (137), où le nom de la substance mesurée prend la forme déterminée et précède l'indication de la mesure, comme si le terme exprimant la mesure était la tête d'une construction génitivale avec le nom de la substance comme dépendant.

\begin{tabular}{|l|l|l|l|l|l|l|}
\hline (137) & $\eta$ & donto & ñoo & muudu & fúla & la! \\
\hline & 1 sg & prêter & mil.D & boisseau & deux & OBL \\
\hline \multicolumn{6}{|c|}{ 'Prête-moi deux boisseaux de mil !' } \\
\hline
\end{tabular}




\section{NOTES}

1. On a de même labay 'terminer' et 'dernier'.

\section{AUTEUR}

DENIS CREISSELS

Université de Lyon

Denis.Creissels@univ-lyon2.fr 